We believe ${ }^{1}$ that some if not most cases of this condition belong to the group of collagen disorders, many probably being connected in some way with rheumatoid disease. We are unconvinced by the arguments advanced that an alteration of nomenclature at this juncture for a condition whose aetiology remains obscure is likely to be of any advantage.-We are, etc.,

RICHARD WARD.

RANDAL STALKER.

Royal Infirmary,

REFERENCE

${ }^{1}$ Ward, R., and Stalker, R., Lancet, 1964, 2, 477

\section{Oral Contraceptives and Breast Cancer}

SIR,-May I reply to Mr. J. J. Shipman's letter (5 September, p. 629) ? Referring to the use of oral contraceptives in the presence of carcinoma of the breast, I would not agree that their use should be " discontinued if carcinoma of the breast is thought or known to be present." Consideration must be given to the possible outcome of not taking the contraceptive drug. The obvious risk is pregnancy, and pregnancy is known to have a serious effect on the progress of some breast carcinomas. The oral contraceptives are in high demand because they have practical application. Other forms of contraceptive fail only too frequently because a natural urge and an available contraceptive do not coincide. The need to avoid pregnancy should be considered against the possible effect of hormones administered orally on a carcinoma of breast, and I believe that the risks of pregnancy are greater.

An alternative method of management is by sterilization associated with bilateral salpingooophorectomy, but few people accept this as a realistic approach.-I am, etc.,

Bangor, Carns.

GARETh Lloyd.

\section{Protection Against Tetanus}

SIR,-In the valuable and concise statement by the Ministry of Health giving the recommendations of the Advisory Group on prutection against tetanus (25 July, p. 243) there is one point which would seem to need clarification. It rather infers that when antitoxin is given an injection of adsorbed tetanus toxoid should be given simultaneously. Can one be certain that the passive immunity thus procured will not interfere with the development of active immunization? Would it not be better to arrange for a course of tetanus toxoid to commence, say, one month later?

Perhaps Dr. H. J. Parish or one of the other authorities would clear up this point, about which some of us are, are present, in doubt.-I am, etc.,

London N.W.7.

A. H. Morley.

\section{Prophylactic Eye-drops}

SIR,-In the B.M.F. (4 July, p. 47) there was an article on failure to control the venereal diseases. In the same issue (Obstetrics in General Practice-Care of the
Newborn Infant, p. 36) under Care of the Eyes I read: "Unless there is a maternal history of vaginal discharge during pregnancy or evidence of discharge during labour, routine prophylactic eye-drops are unnecessary."

I hope Professor Gaisford will think again on this statement. Surely it is a recognized fact to-day that many patients suffering from either syphilis or gonorrhoea, whether male or female, show no obvious cliniçal signs and do not complain of symptoms. At the V.D. clinics I used to see a considerable percentage of cases of gonorrhoea in women who had no symptoms and who showed no obvious clinical signs and yet films or cultures from cervix, urethra, or rectum showed gonococci. The same applies to streptococci and coliform bacilli. I would here like to emphasize the importance of cultures : gonococci can frequently be found in cultures and not in films, and I fear that many laboratories are not very expert in this investigation.

At the Birmingham Eye Hospital, which I used to attend, the number of cases of ophthalmia neonatorum was often in the region of 700 per annum, only a small percentage being gonococcal. I suggest that this considerable number is due either to lack of prophylactic eye-drops or to the failure to instil the drops into the conjunctival sac properly, as I cannot remember a single case of ophthalmia neonatorum occurring in the V.D. maternity ward, where we dealt with some 50 or more midwifery cases a year and where eye-drops were used as routine.

It is, of course, essential that the drops should be non-irritating as well as antiseptic. In any event I cannot see that the eye-drops can do any harm, and in my opinion they should always be used in case there is infection.

Blindness and corneal opacity caused by ophthalmia neonatorum is a tragedy, and if it can be prevented by the simple instillation of a few drops at the time of birth I contend that this should always be done.-I am, etc.,

\section{Stratford-on-Avon, \\ ERIC Assinder. Warwickshire.}

\section{Is Arterial Puncture Dangerous?}

SIR,-In reply to Professor W. W. Mushin's letter (1 August, p. 310), I should like to instance three complications that I have seen following arterial puncture in the last three years.

Firstly, a baby of 9 months, who very nearly bled to death after a femoral artery puncture for cardiac catheterization. The bleeding was discovered in this instance some half an hour after the child had been returned to its cot, ostensibly well, following its cardiac catheterization and left ventriculogram.

The second instance is of a middle-aged lady with valvular disease of the heart, who had a femoral-artery puncture for a left ventriculogram. She subsequently developed a haematoma immediately after the operation, which in the next three weeks grew in size, unchecked, to such an extent that the skin overlying a part of the haematoma became gangrenous. The haematoma itself became infected, and the femoral artery below the haematoma burst, very nearly causing loss of the patient's life. It will be realized that with a massive haematoma in the groin, as in this instance, control of the femoral artery after it had burst was exceedingly difficult.

Thirdly, it is common knowledge that the femoral-artery puncture in small children is frequently accompanied by arterial spasm, and I have seen this last with blanching of the leg for upwards of 25-30 minutes.

Bleeding, following puncture of a highpressure vessel, can and does occur, but I am sure that this complication could be numerically reduced by greater vigilance, less haste, and limitation of the technique to fewer persons. I would be interested to hear of any arterial spasm in babies, of sufficient duration to cause anxiety with regard to limb ischaemia.-I am, etc.,

Queen Elizabeth Hospital,
Birmingham 15.

BRYAN WALDRON.

\section{Early Discharge of Maternity Patients}

SIR,-With reference to the leading article on the early discharge of maternity patients (11 July, p. 70) we would like to offer for comparison the obstetrical statistics of Brookhaven Memorial Hospital, Patchogue, New York. From July 1963 to June 19641,947 deliveries were performed in the 30-bed maternity suite. This is a turnover of approximately 65 patients per bed per annum. The average length of stay was 3.74 days. The average length of stay of vaginal deliveries was 3.48 days; the average length of stay for caesarean deliveries was 7.6 days. There was a total of 109 caesarean deliveries. This length of hospital confinement is about the average for this part of the United States. It should be noted the cases are completely unselected and that all deliveries are performed in hospitals, not in patients' homes.

Although the Obstetrical Flying Squad was a good thing in its day, it is now completely out-dated. The emergencies dealt with by such a team could have been handled much more efficiently if the patient had been in the hospital during her labour. By using beds realistically room can be made to accommodate the mothers who, because of shortage of space, are forced to accept delivery at home.

It should be realized that the hospital is the place for all deliveries, and that domiciliary obstetrics is now completely out-dated. However, it should not be forgotten that the purpose of the hospital is to provide medical care, not hotel accommodation.-We are, etc.,

MARKS G. JACOBY.

James Russell Clark.

Brookhaven Memorial Hospital,

Patchogue, N.Y., U.S.A.

\section{Hazards of Groundnuts}

SIR,-The reccgnition of a new disease responsible for the dcath of many turkeys in $1960^{1}$; the discovery that this resulted from the consumption of groundnut meal contaminated with the fungus Aspergillus flavus, ${ }^{2}$ and the establishment that aflatoxin from this fungus caused carcinoma of the liver in rats $^{3}$ in 1961 ; the detailed study of the chemistry of aflatoxin ${ }^{4}$ and the dissemination of criteria to help exclude infected groundnuts from animal feeds ${ }^{5}$ in 1962; and finally the elucidation of the structure of the two major toxic metabolites ${ }^{8}$ and the 